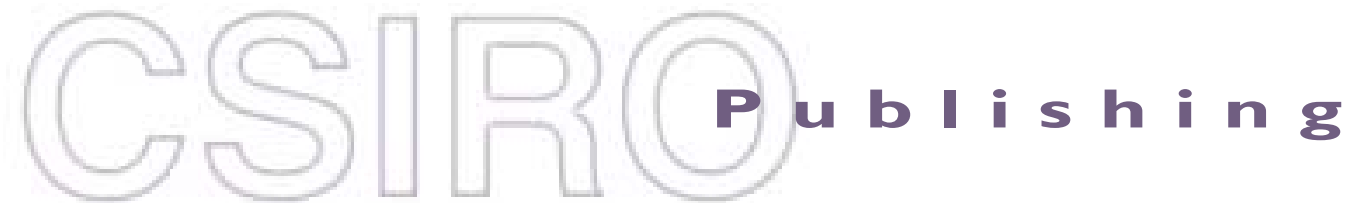

\section{Publications of the Astronomical Society of Australia}

Volume 19, 2002

(C) Astronomical Society of Australia 2002

An international journal of astronomy and astrophysics

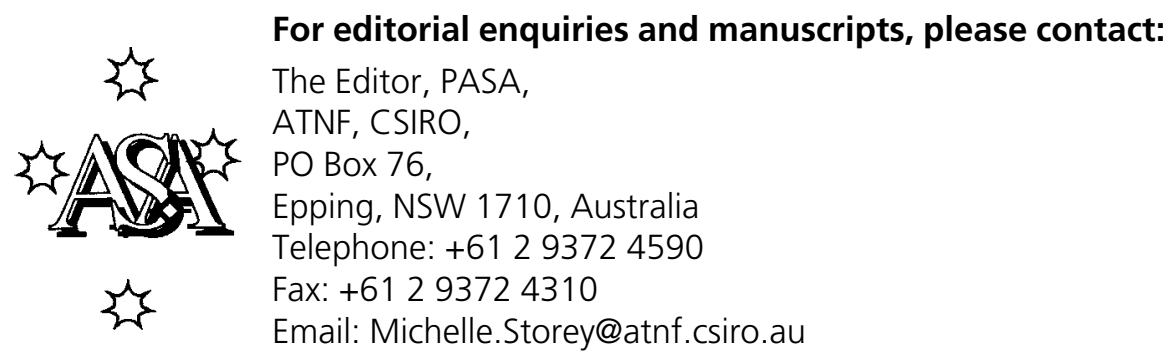

For general enquiries and subscriptions, please contact: CSIRO Publishing PO Box 1139 (150 Oxford St)

Collingwood, Vic. 3066, Australia

Telephone: +6139662 7666

Fax: +61 396627555

Email: publishing.pasa@csiro.au

C S I RO

PUBLISHING Published by CSIRO Publishing

for the Astronomical Society of Australia

www.publish.csiro.au/journals/pasa 


\title{
Polarisation Characteristics and Variability of the Compact Radio Structures of AGN
}

\author{
Denise C. Gabuzda* \\ JIVE, Postbus 2, 7990 AA Dwingeloo, The Netherlands, and \\ Astro Space Centre, Lebedev Physical Institute, Moscow, Russia \\ Received 2001 August 17, accepted 2002 March 25
}

\begin{abstract}
Very long baseline interferometry (VLBI) polarisation measurements provide information about the parsec-scale magnetic field structures in compact active galactic nuclei (AGN), as well as the densities of relativistic and thermal electrons in the radio emitting regions. This paper reviews the role of polarisation VLBI in studies of AGN variability on both relatively long and short (intraday) timescales.
\end{abstract}

Keywords: galaxies: active — galaxies: jets — magnetic fields — polarisation

\section{Parsec-scale Magnetic Fields in Compact AGN}

One of the earliest results to come out of the first VLBI polarisation studies is that different types of compact radio-loud AGN have different characteristic VLBI polarisation structures, with quasars tending to have jets dominated by longitudinal magnetic fields and BL Lac objects tending to have jets dominated by transverse fields (Gabuzda et al. 1989; Cawthorne et al. 1993). This result has stood the test of time, as data have been obtained more systematically and for more complete samples of objects (Gabuzda, Pushkarev, \& Cawthorne 2000d).

Usually, the characteristic transverse jet B field structures of BL Lac objects have been interpreted as evidence for the presence of relativistic shocks that enhance the magnetic field component transverse to the direction of compression (Laing 1980). The dominance of longitudinal B fields in quasar VLBI jets could reflect the effect of shear with the surrounding medium as the jets propagate from the VLBI core; this seems plausible, especially with the discovery of a number of sources that appear to show signs of shear interactions (e.g. 1055-018: Attridge, Roberts, \& Wardle 1999; 0820+225: Gabuzda, Pushkarev, \& Garnich 2001; 0745+241: Pushkarev \& Gabuzda 2002).

Another piece to the puzzle of the relationship between these two types of compact radio-loud AGN is that the apparent superluminal speeds observed in the jets of BL Lac objects on the scales probed by centimetre wavelength VLBI appear to be systematically lower than those observed in core-dominated quasars (Gabuzda et al. 1994, 2000d; Britzen et al. 2001), although there is appreciable overlap between the two distributions and variety in the behaviour displayed by objects in the two classes (e.g. Jorstad et al. 2001). This difference was not noted by Vermeulen \& Cohen (1994), however considerably more speeds for BL Lac objects were included in the later analysis of Gabuzda et al. (2000d). In principle, such a difference in superluminal speeds could imply systematic

\footnotetext{
* Present address: Physics Department, University College Cork, Cork, Ireland. Email gabuzda@phys.ucc.ie
}

differences in either the intrinsic jet flow speeds or the angles of the jets to the line of sight; however, given the absence of convincing evidence that BL Lac objects are more highly beamed than quasars (e.g. Ghisellini et al. 1993), it is most likely that the observed differences in superluminal speeds reflect differences in the intrinsic flow speeds. This seems natural given the identification of most BL Lac objects with FR I rather than more powerful FR II sources (Browne 1983; Wardle, Moore, \& Angel 1984). An interesting question that remains to be explored is whether relatively 'fast' BL Lac objects ('slow' quasars) are more likely to have longitudinal (transverse) jet $\mathbf{B}$ fields on parsec scales and FR II (FR I) arcsecond-scale structures.

It is obviously of interest to try to tie in the systematically different $\mathbf{B}$ field structures and superluminal speeds observed in the VLBI jets of BL Lac objects and quasars. At face value, the difference in the jet $\mathbf{B}$ field structures appears to suggest that shocks are more common in BL Lac object jets; however, the existence of a dominant longitudinal $\mathbf{B}$ field does not preclude the presence of shocks. If there is a strong underlying longitudinal $\mathbf{B}$ field, the net $\mathbf{B}$ can remain longitudinal even when the transverse field from a shock is superimposed. This led to the suggestion that shocks might form in the VLBI jets of both BL Lac objects and quasars, but that the underlying jet B fields in BL Lac objects were predominantly tangled, while those in quasars were predominantly longitudinal, consistent with the idea that the slower jets of BL Lac objects were more turbulent (Duncan \& Hughes 1994; Gabuzda et al. 1994).

More recently, however, the idea that essentially all transverse $\mathbf{B}$ fields on parsec scales are associated with relativistic shocks has been brought into question. There is growing evidence that many of the observed transverse fields correspond to the dominant toroidal component of helical $\mathbf{B}$ fields associated with the VLBI jets. One of the strongest pieces of evidence for this is the discovery of a number of BL Lac objects in which the $\mathbf{B}$ fields remain transverse all along the jets, even in the presence 
of appreciable bending (Gabuzda 1999; Pushkarev \& Gabuzda 2000; Gabuzda \& Pushkarev 2002; see Figure 1). A 'conventional' interpretation in the context of shock models would require the presence of a whole series of relativistic shocks along the jet, each of which enhances the local transverse B field (Figure 2, left). Although theoretically possible, this picture seems contrived. If the observed transverse $\mathbf{B}$ fields in these sources are associated with the dominant toroidal component of helical jet $\mathbf{B}$ fields, maintenance of the transverse field orientation as the jets curve would be 'built in' (Figure 2, right). In addition, this type of $\mathbf{B}$-field structure is predicted by many theoretical

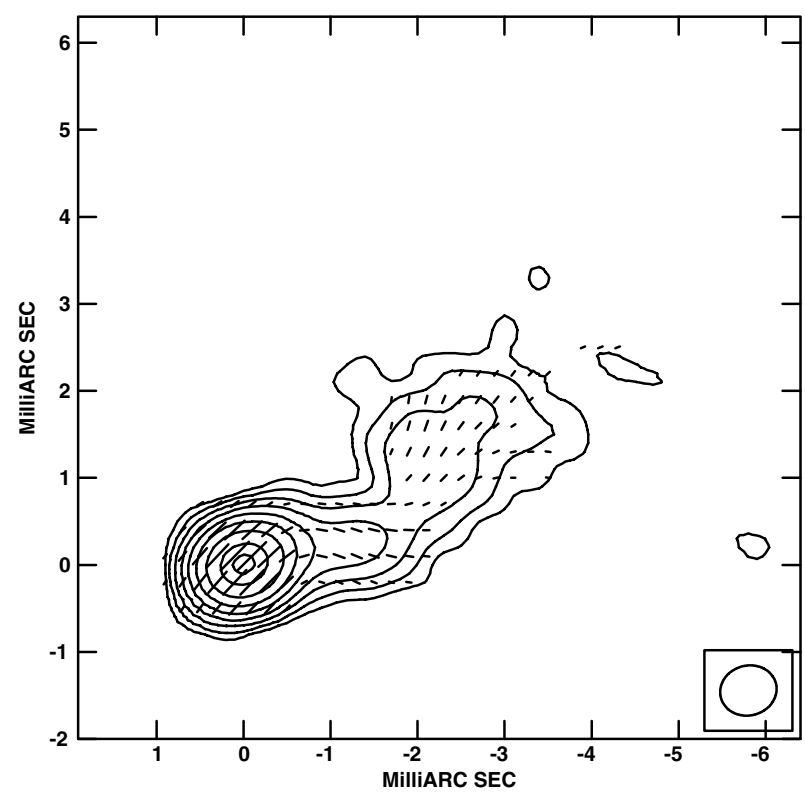

Figure $115 \mathrm{GHz}$ total intensity contours showing the very curved VLBI jet of $1749+701$ with observed $8 \mathrm{GHz}$ polarisation electric vectors superimposed. The vectors are aligned with the jet and follow it as it bends, so that the magnetic field in the optically thin jet is everywhere transverse. models for jet formation and propagation, such as those in which the magnetic field acquires a dominant toroidal component due to rotation of the central accreting object (e.g. Ustyugova et al. 2000; Nakamura, Uchida, \& Hirose 2001) or a toroidal field component develops as a consequence of the currents flowing in the jet (Istomin \& Pariev 1996).

Thus, it is likely that many of the transverse $\mathbf{B}$ fields that have previously been ascribed to shocks are actually associated with a toroidal jet B-field component. Several candidates have been found among BL Lac objects, but it may be that toroidal/helical jet $\mathbf{B}$ fields are characteristic of other types of AGN as well. One interesting possibility is that both BL Lac objects and quasars have helical jet $\mathbf{B}$ fields, but that the slower flow speeds in BL Lac objects lead to an increased pitch angle for the helical field, increasing the dominance of the toroidal field component. Of course, some bright, compact features in the VLBI jets of AGN with transverse B fields probably are associated with shocks. However, it is clearly inappropriate to interpret all features with transverse B fields as shocks, since many of them probably reflect the dominant orientation of the underlying jet $\mathbf{B}$ fields.

\section{Polarisation VLBI Studies of Intraday Variability}

The phenomenon of intraday variability, or IDV, is well established, but remains incompletely understood (Wagner \& Witzel 1995). Our own approach to studying IDV has been simple and direct: to obtain VLBI polarisation data simultaneously with integrated polarisation measurements, then construct independent VLBI polarisation images for every few hours of data, in order to determine whether rapid variability occurred on milliarcsecond scales and if it was confined to a single region in the VLBI structure.

The most burning and controversial question connected with IDV is what fractions of intraday variations are
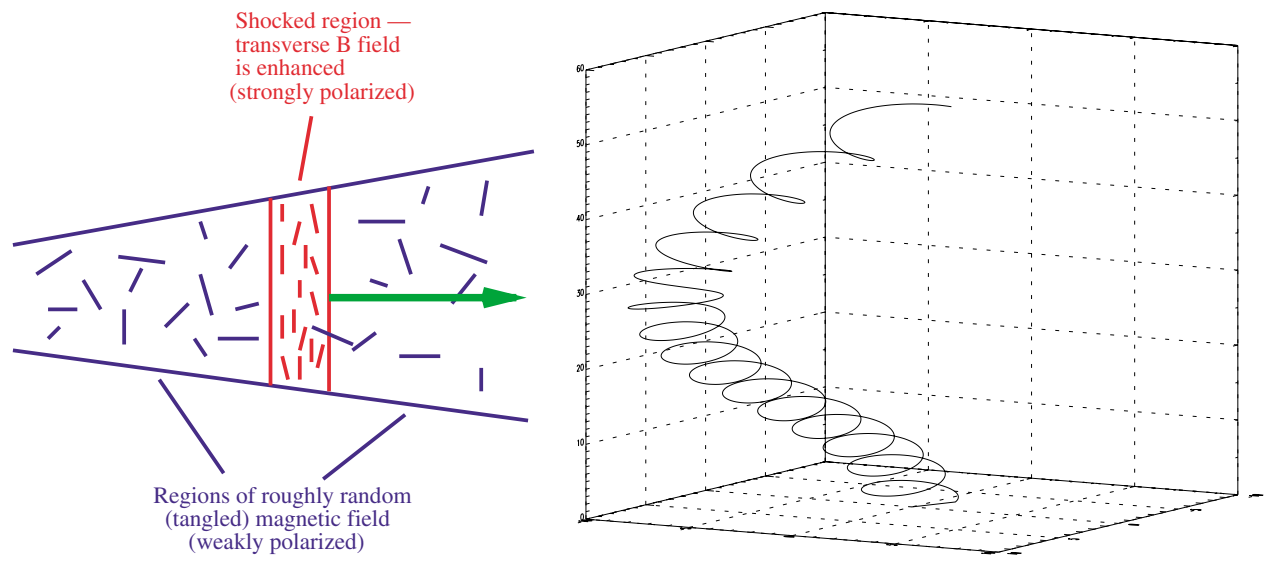

Figure 2 Left: Schematic of shock mechanism for amplification of transverse B field component; to explain extended jet regions dominated by transverse $\mathbf{B}$ fields, this mechanism would require a series of many shocks, each of which enhances the local transverse field component. Right: Schematic of a helical B field twisted around a curved jet, as might occur, for example, if the field is 'wound up' by rotation. In this case, maintenance of the transverse field orientation everywhere along the jet is 'built in', making it an attractive alternative to the shock model for jets with extended regions with transverse B fields (see Figure 1). 
intrinsic and extrinsic (e.g. Rickett et al. 1995). A number of spectacular IDV events were almost certainly due to refractive scintillation in the interstellar medium (Kedziora-Chudczer et al. 1998, 2000; Dennett-Thorpe \& de Bruyn 2000). At the same time, a number of observations, including our own polarisation VLBI observations, indicate that at least some IDV is dominated by an intrinsic component. It is not uncommon to observe appreciable IDV in polarisation without detectable variations in the total intensity (Gabuzda et al. 2000a,b; Gabuzda, Kochenov, \& Cawthorne 2000c; Kedziora-Chudczer et al. 2001), which cannot be easily explained in plausible models without an intrinsic component to the variability. In addition, there may be evidence for correlated IDV in the optical and radio, which cannot be accommodated by scintillation models (Quirrenbach et al. 1991).

Thus far, we have VLBI polarisation results for six sources (Gabuzda et al. 2000a,b,c).

In several cases, rapid (timescales of several hours) polarisation variations occurred in the VLBI core; in 2155-152, only one of two VLBI components separated by about 4-5 mas varied, placing constraints on models for extrinsic variability. For example, given that the polarised emission of the two features is comparably compact, is it reasonable for the properties of the scattering screen to be appreciably different for the lines of sight to these two closely spaced features, such that one would scintillate while the other would not? Most surprising was the discovery in $0716+714$ of dramatic polarisation variations on scales of tens of milliarcseconds: previously, no-one had suspected that such compact features could be present so far from the VLBI core. In this case, it may be difficult to understand why this distant feature should scintillate when the compact core did not, unless the scattering screen is inhomogeneous on scales of tens of milliarcseconds.

The fact that polarisation IDV is sometimes observed in the absence of detectable total intensity IDV provides some of the strongest evidence that there is an appreciable intrinsic component to some IDV events. Scintillation models must be able to explain such variations if it is to be claimed that all IDV can be understood as extrinsic variations. It remains to be seen if plausible scintillation models capable of giving rise to such variations can be developed.

At the same time, the nature of possible intrinsic mechanisms for IDV also remains unclear. Our result for $0716+714$ requires that IDV mechanisms be able to operate on a wide range of scales, suggesting that some IDV may be associated with interactions between compact regions in the relativistic jets and their surrounding medium. We should also recall that the high brightness temperatures implied by IDV events present a problem only if such events continue for too long - the inverse Compton limit can be exceeded briefly, after which the electrons rapidly cool to brightness temperatures near or below the limit (Kellermann \& Pauliny-Toth 1969). Thus, it may be that we have been neglecting an obvious intrinsic mechanism for IDV: temporary excursions of the brightness temperature above the inverse Compton limit. Another old idea that has recently been neglected is the fact that, in some optically thick regimes, rapid changes in optical depth can lead to dramatic changes in polarisation without appreciable changes in total intensity (Aller 1970; Gabuzda, Wardle, \& Roberts 1989).

\section{Faraday Rotation Measurements and Rotation Measure Variability}

As VLBI polarimetry matures as an observing technique, more and more parsec-scale Faraday rotation measurements are becoming available. These offer the first direct information about the thermal plasma in the vicinity of the VLBI jets, and have provided some surprises.

Among the earliest such reports were made by Taylor (1998, 2000), who found enhanced rotation measures in the VLBI core regions of seven of eight bright quasars; in all cases, these sources have small integrated rotation measures, thought to have been consistent with a Galactic origin. The absolute values of these core rotation measures range from 1200 to more than $2000 \mathrm{rad} \mathrm{m}^{-2}$, while the typical values in the VLBI jets are less than $100 \mathrm{rad} \mathrm{m}^{-2}$, sometimes consistent with $0 \mathrm{rad} \mathrm{m}^{-2}$. More recently, Taylor (2000) and Zavala \& Taylor (2001) have reported variability in the core-region rotation measures of 3 C273 and 3C279.

Nan et al. (1999) have presented intriguing observations of the parsec-scale rotation-measure distribution in the compact steep spectrum source 3C119. Their observations reveal a concentration of thermal plasma near a bright jet component far from the core, near a sharp bend in the VLBI jet. They also detected a gradient in the rotation measure, which increases toward the leading edge of this component (i.e. decreases back towards the core). Nan et al. (1999) concluded that their observations pointed toward a collision between the jet and a dense cloud at the location of this bright jet component, which deflected the VLBI jet. One somewhat surprising recent result is the detection of non-uniform rotation-measure distributions in a number of BL Lac objects as well. Reynolds, Cawthorne, \& Gabuzda (2001) found an enhanced rotation measure near the VLBI core of BL Lac, and also evidence that the observed core rotation measure is variable. Gabuzda \& Chernetsky (2002) likewise detected an enhancement in the parsec-scale rotation measure of $1803+784$ in the vicinity of the VLBI core, which seems to drop off fairly smoothly with distance from the core, similar to the behaviour seen in some of the quasars studied by Taylor (1998, 2000). Finally, Gabuzda et al. (2001) detected a concentration of rotation measure well separated from the VLBI core of $0820+225$, near a fairly sharp bend in the VLBI jet (Figure 3); as in the case of 3C119 (Nan et al. 1999), it is tempting to think that this reflects some interaction between the jet and a cloud or clump in the surrounding medium.

Two notes are relevant in connection with these collected results. First, it is interesting that non-uniform parsec-scale rotation-measure distributions have been 

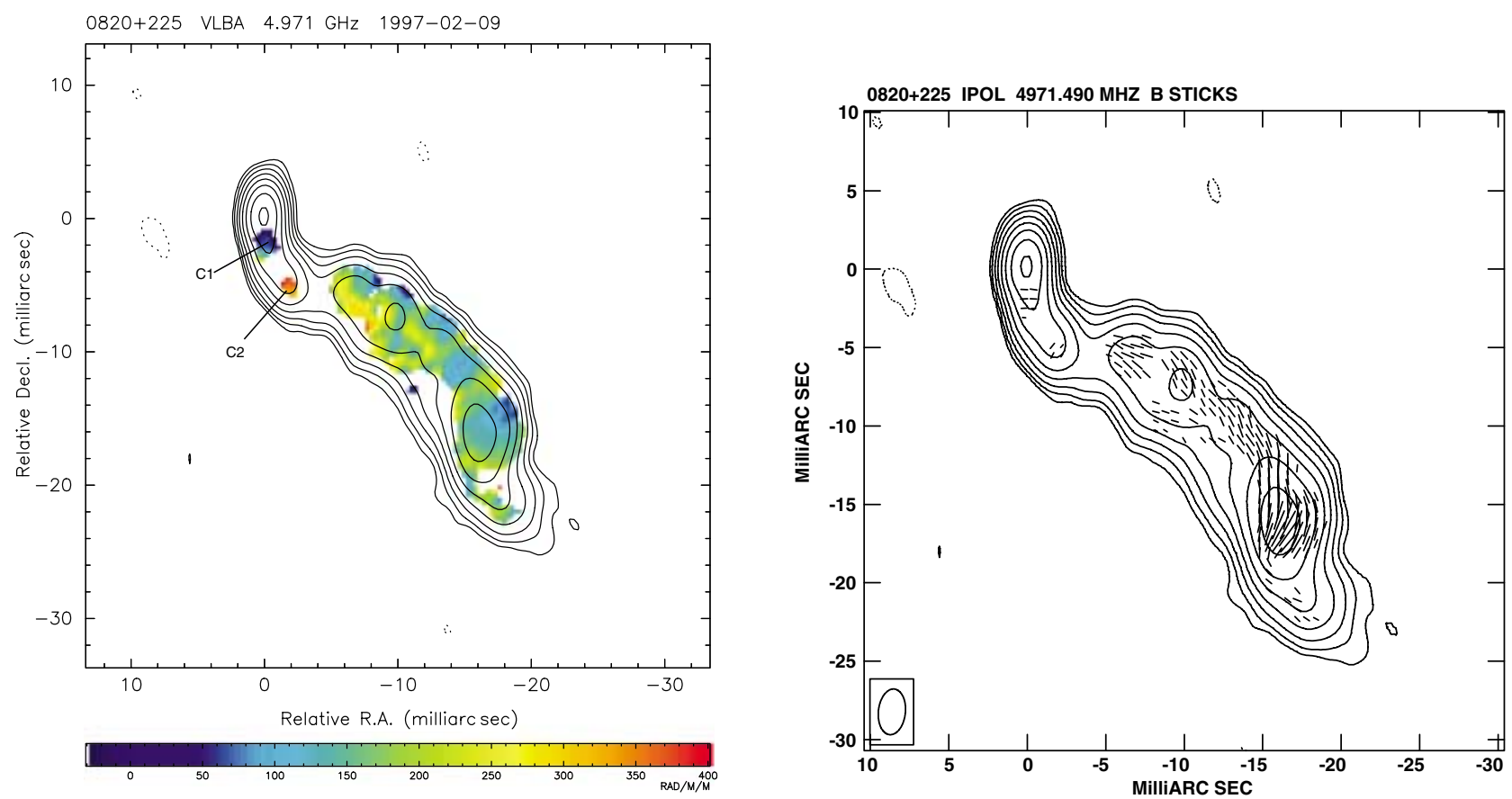

Figure 3 Left: $5 \mathrm{GHz}$ total intensity contours showing the VLBI jet of the BL Lac object $0820+225$ with the observed rotation-measure distribution superimposed in colour. The rotation measure in the inner knot $\mathrm{C} 1$ is only $\simeq 40 \mathrm{rad} \mathrm{m}^{-2}$, but grows to $\simeq 350 \mathrm{rad} \mathrm{m}^{-2}$ at the knot C2. Right: magnetic-field structure in the VLBI jet after correction for the non-uniform rotation-measure distribution. The $\mathbf{B}$ field in the inner part of the jet is transverse, and becomes longitudinal after the first bend, suggestive of a shear interaction (see Gabuzda et al. 2001).

detected in several BL Lac objects as well as quasars, which have much stronger optical lines. This may suggest that the relatively low luminosity of the optical line emission in BL Lac objects is not associated purely with a dearth of gas in these sources, and that another factor is required, such as a lower level of ionising continuum flux. The formerly popular idea that the optical lines of BL Lac objects might just be 'swamped' by their highly beamed optical continua does not work: there is no convincing evidence that BL Lac objects are more highly beamed than quasars (Ghisellini et al. 1993) and studies have indicated that the actual luminosities of the lines in BL Lac objects are systematically lower than those in quasars (Padovani 1992). In the context of unification schemes, this is consistent with the analysis of Baum, Zirbel, \& O'Dea (1995), who found that the optical emission lines of FR I radio galaxies are systematically less luminous than those of FR II radio galaxies of the same total radio luminosity or radio core power, and concluded that this was most likely associated with a lack of ionising UV continuum in FR I sources.

Second, it is important to realise that variability in parsec-scale rotation measures does not necessarily reflect variations in the distribution of thermal plasma or line-ofsight magnetic field in the region surrounding the radio core and jet. It is probably more likely that the observed variable rotation measures occur due to the passage of unresolved polarised components behind a relatively static foreground plasma screen in the vicinity of the radio source. In this way, VLBI rotation-measure variability studies may potentially provide a powerful tool for probing both the compact radio structures and the distribution of thermal plasma surrounding them with higher resolution than achieved directly with the VLBI arrays used.

\section{Summary}

It is now well established that different types of compact radio-loud AGN have different characteristic VLBI polarisation structures, with quasars tending to have jets dominated by longitudinal $\mathbf{B}$ fields and BL Lac objects tending to have jets dominated by transverse fields. Although this systematic difference was initially interpreted as evidence that transverse shocks were more common in the jets of BL Lac objects, another possibility that has arisen more recently is that this corresponds to dominance of the longitudinal or toroidal component of helical jet magnetic fields. In addition, the relativistic jets of these two types of sources have different characteristic superluminal speeds, with those in quasars systematically higher than those in BL Lac objects. These differences in the typical $\mathbf{B}$ field structures and VLBI jet properties of these two types of compact, highly polarised AGN - distinguished primarily by differences in the strength of their optical line emission - appear to reflect physical interconnections between the optical and VLBI properties of AGN, whose nature is not yet clear.

Intraday variability in both total and polarised flux density is observed in a substantial number of compact AGN. The most extreme variations are almost certainly dominated by scintillation, but it remains likely that there is an appreciable contribution from intrinsic variations in some 
cases. Analyses of VLBI polarisation observations made during IDV events have made it possible to identify specific milliarcsecond-scale varying components and isolate their properties, providing unique information about the nature of the IDV, and probably demonstrating the necessity of a dominant intrinsic component to the variability in some cases. Such work clearly has potential for distinguishing IDV events dominated by scintillation from those dominated by intrinsic variability, and can be expected to provide critical information for IDV models.

Finally, one very recent development in VLBI polarisation studies is the discovery of a number of AGN with non-uniform distributions of rotation measure (thermal plasma) in the immediate vicinity of their VLBI jets. It is interesting that non-uniform thermal-plasma distributions have been detected in a number of BL Lac objects as well as quasars. The observed rotation measures near the VLBI cores of several sources have been found to change with time. This is probably associated with the passage of polarised jet components behind different parts of a relatively static foreground plasma screen, and therefore may provide a powerful new tool for studies of the thermal plasma surrounding the compact radio structures of AGN on parsec scales.

\section{Acknowledgments}

The author acknowledges support from the European Commission under the IHP Programme (ARI) contract No. HPRI-CT-1999-00045.

\section{References}

Aller, H. D. 1970, ApJ, 161, 1

Attridge, J. M., Roberts, D. H., \& Wardle, J. F. C. 1999, ApJ, 518, L87

Baum, S. A., Zirbel, E. L., \& O’Dea, C. P. 1995, ApJ, 451, 88

Britzen, S., et al. 2001, in Galaxies and Their Constituents at the Highest Angular Resolutions, IAU Symposium 205, ASP Conf. Series, eds R. T. Schilizzi, S. N. Vogel, F. Paresce, \& M. S. Elvis (San Francisco: ASP), 106

Browne, I. W. A. 1983, MNRAS, 204, 23

Cawthorne, T. V., et al. 1993, ApJ, 416, 496

Dennett-Thorpe, J., \& de Bruyn, A. G. 2000, ApJ, 529, L65

Duncan, G. C., \& Hughes, P. A. 1994, ApJ, 436, 119

Gabuzda, D. C. 1999, NewAR, 43, 695

Gabuzda, D. C., \& Chernetsky, V. A. 2002, MNRAS, in press

Gabuzda, D. C., Cawthorne, T. V., Roberts, D. H., \& Wardle, J. F. C. 1989, ApJ, 347, 701

Gabuzda, D. C., Kochenov, P. Yu., Cawthorne, T. V., \& Kollgaard, R. I. 2000a, MNRAS, 313, 627

Gabuzda, D. C., Kochenov, P. Yu., Kollgaard, R. I., \& Cawthorne, T. V. 2000b, MNRAS, 315, 229
Gabuzda, D. C., Kochenov, P. Yu., \& Cawthorne, T. V. 2000c, MNRAS, 319, 1125

Gabuzda, D. C., Mullan, C. M., Cawthorne, T. V., Wardle, J. F. C., \& Roberts, D. H. 1994, ApJ, 435, 140

Gabuzda, D. C., \& Pushkarev, A. B. 2002, in Particles and Fields in Radio Galaxies, ASP Conf. Series 250, eds R. Laing, \& K. Blundell (San Francisco: ASP), 180 (in press)

Gabuzda, D. C., Pushkarev, A. B., \& Cawthorne, T. V. 2000d, MNRAS, 319, 1109

Gabuzda, D. C., Pushkarev, A. B., \& Garnich, N. N. 2001, MNRAS, 327,1

Gabuzda, D. C., Wardle, J. F. C., \& Roberts, D. H. 1989, ApJ, 338, 743

Ghisellini, G., Padovani, P., Celotti, A., \& Maraschi, L. 1993, ApJ, 407, 65

Istomin, Ya. N., \& Pariev, V. I. 1996, MNRAS, 281, 1

Jorstad, S. G., Marscher, A. P., Mattox, J. R., Wehrle, A. E., Bloom, S. D., \& Yurchenko, A. V. 2001, ApJS, 134, 181

Kedziora-Chudczer, L. L., Jauncey, D. L., Weiginga, M. H., Tzioumis, A. K., \& Reynolds, J. E. 2001, MNRAS, 325, 1411

Kedziora-Chudczer, L. L., Marquart, J.-P., Jauncey, D. L., \& Rayner, D. P. 2000, in Astrophysical Phenomena Revealed by Space VLBI, eds H. Hirabayashi, P. G. Edwards, \& D. W. Murphy (Tokyo: ISAS), 143

Kedziora-Chudczer, L. L., Walker, M. A., Jauncey, D. L., Wieringa, M. H., Reynolds, J. E., Tzioumis, A. K., \& Nicolson, G. D. 1998, in IAU Colloquium 164: Radio Emission from Galactic and Extragalactic Compact Sources, eds J. A. Zensus, G. B. Taylor, \& J. M. Wrobel, ASP Conf. Series 144 (San Francisco: ASP), 267

Kellermann, K. I., \& Pauliny-Toth, I. I. K. 1969, ApJ, 155, L71

Laing, R. 1980, MNRAS, 193, 439

Nakamura, M., Uchida, Y., \& Hirose, S. 2001, NewA, 6, 61

Nan, R., Gabuzda, D. C., Kameno, S., Schilizzi, R. T., \& Inoue, M. 1999, A\&A, 344, 402

Padovani, P. 1992, MNRAS, 257, 404

Pushkarev, A. B., \& Gabuzda, D. C. 2000, in Proceedings of the Fifth EVN Symposium, eds J. Conway, A. Polatidis, \& R. Booth (Gothenburg: Onsala Space Observatory, Chalmers Technical University), 63

Pushkarev, A. B., \& Gabuzda, D. C. 2002, in Particles and Fields in Radio Galaxies, ASP Conf. Series 250, eds R. Laing, \& K. Blundell (San Francisco: ASP), 200 (in press)

Quirrenbach, A., et al. 1991, ApJ, 372, L71

Reynolds, C., Cawthorne, T. V., \& Gabuzda, D. C. 2001, MNRAS, 327,1071

Rickett, B. J., Quirrenbach, A., Witzel, A., Wegner, R., \& Krichbaum, T. P. 1995, A\&A, 293, 479

Taylor, G. B. 1998, ApJ, 506, 637

Taylor, G. B. 2000, ApJ, 533, 95

Ustyugova, G. V., Lovelace, R. V. E., Romanova, M. M., Li, H., \& Colgate, S. A. 2000, ApJ, 541, L21

Vermeulen, R. C., \& Cohen, M. H. 1994, ApJ, 430, 467

Wagner, S. J., \& Witzel, A. 1995, ARA\&A, 33, 163

Wardle, J. F. C., Moore, R. L., \& Angel, J. R. P. 1984, ApJ, 279, 93

Zavala, R. T., \& Taylor, G. B. 2001, ApJ, 550, L147 